\title{
Terrestrial and Astronomical Sources of Circular Polarisation: A Fresh Look at the Origin of OF Homochirality on Earth
}

\author{
Ramon D. Wolstencroft \\ Royal Observatory, Edinburgh EH9 3HJ, U.K.
}

\begin{abstract}
In searching for a terrestrial source of circular polarization that could be an agent for homochirality, I have re-discovered earlier work by Ivanoff \& Waterman (1958) showing that total internal reflection of sunlight scattered by water or suspended particles can, in calm shallow waters such as tidal pools, yield a degree of circular polarization $>10 \%$ in favourable directions. A terrestrial source would have the distinct advantage over an astronomical source that the perilous journey to the planetary surface is eliminated. The relative viability of this mechanism is compared with the widely discussed astronomical mechanism of Bailey et al. (1998).
\end{abstract}

\section{Introduction}

The key molecules of biochemistry, such as proteins and polysaccharides, are assembled from chiral monomers of a single handedness. How this initial generation of chiral molecules first arose on Earth is a long-standing puzzle that has existed since the time of Pasteur. A possible mechanism that has been much discussed recently is one in which a racemic mixture of molecules underwent asymmetric photolysis by the action of an extraterrestrial source of circularly polarized light (CPL), leaving a significant excess of molecules of one handedness. Since the relevant circular dichroism bands are in the ultraviolet (UV) (typically shortward of $300 \mathrm{~nm}$ ) there must be a convincing mechanism for producing appreciable UVCPL.

\subsection{The Astronomical Scenario}

Most of the recent discussion has involved the production of UVCPL via the scattering of starlight by aligned grains in a region of star formation: this UVCPL is thought to have illuminated a second set of grains in the proto-solar nebula which were coated with a racemic mixture of a few key molecules; these grains were shaded from the direct light of the illuminating star so as not to dilute the UVCPL and so permit asymmetric photolysis of these molecules to take place (Bailey et al. 1998; Bailey 2001): at the same time the whole of the proto-solar nebula (likely to be several $100 \mathrm{AU}$ in size) had to see this single sign of UVCPL photolyse its molecules and yet still remain shaded from the direct starlight this puts severe constraints on the geometry involved. These grains and their partially chiral surface molecules were somehow transported to Earth, presumably during an interval measured in $\mathrm{Myr}$, and after finally surviving passage through the atmosphere and probably an ocean landing, they arrived intact 
and without racemization. Although this scenario is feasible in principle, if true it clearly is not a straightforward or highly probable process.

\subsection{The Terrestrial Scenario}

On the face of it a more logical place for this process to have occurred might instead have been on Earth itself, provided there was a source of appreciable UVCPL at or before the epoch about 3.8 Byr BP when life on Earth may have begun. Since the atmosphere contained no $\mathrm{O}_{2}$ or $\mathrm{O}_{3}$ at this time, the solar UV could readily reach the surface down to wavelengths of about $230 \mathrm{~nm}$ (limited shortward of this by $\mathrm{CO}_{2}$ absorption, whose bond energy is $5.52 \mathrm{ev}$ ). The question is whether there was a mechanism to produce UVCPL.

When I first looked at this question (Wolstencroft 1985), I dismissed the idea of any astronomical source of CPL. This was on the grounds that the prospects of any chiral molecules being created by a magnetic white dwarf ,the only obvious class of source (then) with strong CPL emission, passing through a molecular cloud was too unlikely: and in any case how could these chiral molecules safely reach the Earth? Instead I considered the daytime sky as the only viable source of CPL : however I observed only low values of the degree of circular polarization, q (up to $0.5 \%$ at $350 \mathrm{~nm}$ ), roughly in agreement with model computations by Hitzfelder et al (1976). Of course the daytime sky would have had a different and perhaps a higher CPL at that distant epoch. The circular polarization we see today arises as light, partially linearly polarized in a first (Rayleigh) scattering, has undergone a phase change in a second scattering by aerosols, which differs between orthogonal components, and hence leads to a circular component. Thus the CPL tends to increase with decreasing wavelength (as the linearly polarized [Rayleigh] first scattering increases) until multiple scattering starts to dilute the effect. On the early Earth with its atmosphere being relatively UVB transparent, this mechanism might have produced a level of a few percent at wavelengths well below $300 \mathrm{~nm}$ in certain directions. However because the net UVCPL would have been zero when averaged over all sky directions, one needs to envisage some geometrical source of asymmetry, such as a sloping terrain which could have obscured part of the sky, in order to realise a small but viable net UVCPL (Wolstencroft 1985). So the UVCPL of the ancient daytime sky is not a promising candidate.

However a terrestrial mechanism, previously overlooked at the time, is capable of producing appreciable CPL: circular polarization of submarine illumination can be produced by total internal reflection of scattered linearly polarized sunlight in calm shallow waters.

\section{Circular Polarisation of Submarine Illumination}

The state of polarization of natural underwater illumination is mainly linear, but in shallow waters and in certain directions a significant circular component is observed which can become comparable with the linearly polarized component and can exceed $10 \%$. This property of the so-called submarine illumination was first discovered by Waterman (1954) and studied in detail by Ivanoff \& Waterman (1958) (IW). They used a polarimeter comprising a calcite prism and circular polarization analyzer to study the state of polarization (see, Waterman 1955). 
Having carried out observations both at sea and in the laboratory (aquarium) IW concluded that the likely origin of the circular polarization was total internal reflection at the water surface of linearly polarized light, which was itself produced mainly by the scattering of sunlight by water and suspended particles in the water. They developed a model in which the dependence of the level of CPL on the direction of the line of sight and of the Sun could be calculated; they showed that their observations were broadly consistent with this model.

In their paper IW showed that a general (submarine) observer at B, looking along a line of sight intersecting the ocean surface at a point $\mathrm{A}$ and at angle $\mathrm{V}$ to the vertical (see Fig. 1), sees two contributions. One is from light scattered (and hence partially linearly polarized) at points along AB towards B; and the other from light scattered elsewhere (i.e., not on $A B$ ) and totally internally reflected at $\mathrm{A}$ towards the observer (this can only occur at an angle $\mathrm{V}>48.8^{\circ}$, the critical angle for $\mathrm{n}$ (water) $=1.33$ ). This second component is converted from partially linearly polarized to partially circularly polarized light on total internal reflection because a phase difference, $d$, is introduced between the components parallel(l) and perpendicular(r) to the plane of incidence: $d$ depends on $V$, and $\mathrm{d}$ peaks at $32^{\circ}$ when $\mathrm{V}=58^{\circ}$. When the $\mathrm{l}$ and $\mathrm{r}$ components incident at $\mathrm{A}$ are equal, the axial ratio of the polarization ellipse peaks at $b / a=0.30$. This corresponds to an (undiluted) degree of circular polarization $\mathrm{q}($ und $)=2 \mathrm{~T} /(1+\mathrm{T} 2)$, where $\mathrm{T}=\tan (\mathrm{d} / 2)=\tan 16^{\circ}$, ie $\mathrm{q}($ und $)=53 \%$. Supposing that just $30 \%$ of the light was transformed from linear to circular polarization the observed circular would be diluted by unpolarized light to a value $\mathrm{q}(\mathrm{obs})=0.3 \times 53 \%=16 \%$.

The model calculations of IW show the dependence of CPL and hence of $q($ und) on the azimuth and zenith distance of the sun, the line of sight and on the depth of the observer. The CPL is shown to be greatest: (a) when the solar zenith distance $>60^{\circ}$; (b) in a direction $90^{\circ}$ from the solar azimuth; and as expected $q$ (west) =-q(east) for a given azimuth difference west or east of the sun's bearing (basic anti-symmetry); (c) at an angle, $\mathrm{V}$, between the line of sight and the vertical, which changes with depth: $>80^{\circ}$ at the shallowest depths; (d) at very shallow depths measured in $\mathrm{cm}$; (e) when the water surface is calm, i.e. flat.

IW conclude that values of $\mathrm{q}(\mathrm{obs})>10 \%$ apply in favourable conditions, e.g. in calm shallow waters of low turbidity when the sun is low in the sky and the direction of observation is at least $45^{\circ}$ from the solar azimuth. Because of the anti-symmetry of the pattern of CPL across the "submarine sky" (see (b) above) one still needs some bias favouring the morning or evening "sky", however this is more readily accomplished than for the atmospherically scattered daytime sky because the highest values of the degree of circular polarization, q, occur near sunrise or sunset. Furthermore if the line of sight is restricted to the "sky" in the south or north where $\mathrm{q}$ is relatively constant across a wide range of azimuths then a net CPL of a given sign and $q>5 \%$ could result.

Since we also require that $\mathrm{q}(\mathrm{obs})$ be significant in the ultraviolet we need also to address the question of the UV transmission of the atmosphere and ocean, lake or pool. At shallow depths this may not be a problem down to about $230 \mathrm{~nm}$ : a sharp rise in the attenuation coefficient, $\mathrm{k}$ (per $\mathrm{m}$ ), occurs between $300 \mathrm{~nm}(0.15)$ and $230 \mathrm{~nm}$ (0.97) (Smith \& Baker 1981) but the (1/e) penetration path length in water of $1 \mathrm{~m}$ at $230 \mathrm{~nm}$ would be enough to allow the process to operate down to this wavelength, but not much below $230 \mathrm{~nm}$ 


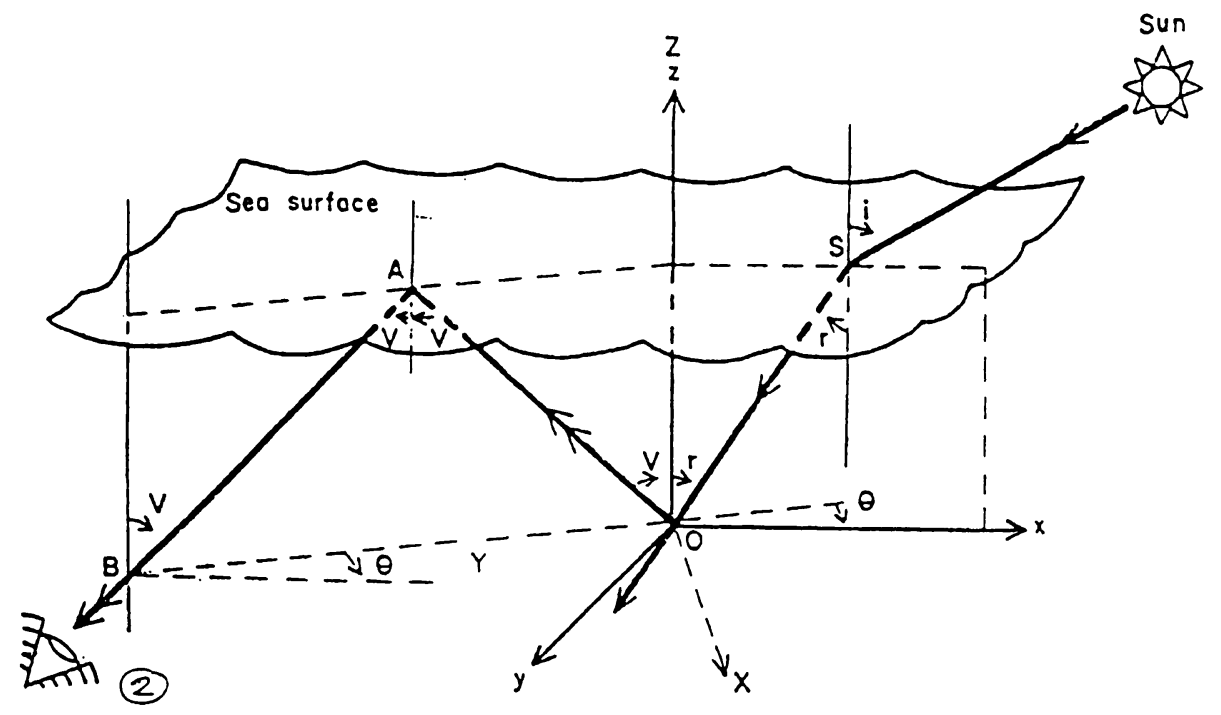

Figure 1. Diagram to illustrate origins of polarised light seen by an observer at $B$ looking in direction $A B$. One component originates in light directly scattered along $A B$, the second from total reflection at $A$ of light scattered along $O A$. The former is linearly polarised while the letter may be elliptically polarised. $i$, solar zenith angle; $r$, refracted angle of sun's rays; $x z$, vertical plane of sunlight incidence parallel to the plane through observer and sun; $Y Z$, vertical plane passing through the line of sight $B A ; x y z$, perpendicular coordinate system relative to the plane of incidence; $X Y Z$, coordinate system relative to the plane of the line of sight; $\theta$, difference angle between $x z$ and $Y Z$ planes; $Y$, angular deviation of the line of sight from the vertical.

where by coincidence the atmospheric carbon dioxide would extinguish any solar UV. Note that although the sun was $30 \%$ cooler than today, enhancement of the solar UV during its T Tauri stage (Canuto et al. 1983) could have made up for any perceived UV deficit relative to today's solar flux.

\section{Discussion}

Although the UVCPL of the Archean daytime sky may have been too small to provide an adequate source for asymmetric photolysis there are much better prospects for UVCPL produced in the surface layers of Archean oceans or tidal pools since the level of UVCPL is considerably higher. Assuming that asymmetric photolysis can take place in this marine situation, perhaps in a quiet, calm and shallow tidal pool or lake, how does it compare with the astronomical mechanism of Bailey et al (1998)? There are several relevant issues, namely (1) dilution of the CPL by starlight; (2) racemization of the chiral products; and (3) the amino acid absorption spectra. Firstly, in the astronomical scenario the direct light from the nearby star, whose light is being scattered to produce CPL, must be shielded from the large proto-solar nebula to prevent dilution of the CPL, which seems a little artificial. In the terrestrial scenario direct sunlight 
would be a problem for a daytime sky source of CPL, but not for a marine source of CPL: direct sunlight cannot enter the water at the range of angles $\left(\mathrm{V}>48.8^{\circ}\right)$ at which total internal reflection occurs and hence cannot directly cause dilution when the water surface is calm, although dilution after scattering will occur as discussed above.

Concerning racemization, the half-lives may be in excess of $1 \mathrm{Myr}$ in cold dry environments, but in the warm $(300 \mathrm{~K})$ and wet conditions likely to have been experienced after splash down in the Archean ocean, the half-life would have been measured in hundreds of years. The extreme temperatures experienced on passage through the terrestrial atmosphere, and on impact at the surface, could in principle have caused racemization in a few minutes (Basiuk 2001). Nevertheless it is worth noting that an enantiomeric excess of amino acids of rare or unknown terrestrial distribution was detected in the Murchison meteorite by Cronin \& Pizzarello (1997) and is attributed to the incomplete racemization of amino acids during a liquid water phase on the parent asteroidal body (Cohen \& Chyba 2000), as well as during passage through the atmosphere. For a terrestrial marine source of CPL the process of asymmetric photolysis would be modified due to aqueous alteration but should still proceed; if this in situ photolysis is intermittent, slow racemization will presumably occur at times, but the net enantiomeric excess should grow provided that the illumination geometry and other factors are favourable and produce a viable UVCPL. We consider the amino acid (AA) absorption spectra. The AA with conjugated aromatic rings absorb at $280 \mathrm{~nm}$ (Trp), $274 \mathrm{~nm}$ (Tyr) and $257 \mathrm{~nm}$ (Phe), all of which would be accessible in the marine situation, but the aliphatic AA absorb in the 190 to $230 \mathrm{~nm}$ range which would thus not be accessible. An astronomical CPL source would not have this difficulty (Bailey 2001). A related question of how a broad band CPL source can be effective when circular dichroism bands typically are narrow and alternate in sign Mason (1997) is addressed by Bailey (2001), who argues that this still yields an effect though with some reduction in effectiveness.

\section{References}

Bailey, J., Chrysostomou, A., Hough, J. H., Gledhill, T. M., McCall, A., Clark, S., Menard, F. \& Tamura, M. 1998, Science, 281, 672

Bailey, J. 2001, Origins of Life and Evolution of the Biosphere, 31, 167

Basiuk, V. A. 2001, Adv. Space Research, 27, 335

Canuto, V. M, et al. 1983, Nature, 305, 281

Cohen, B. A., \& Chyba, C. 2000, Icarus, 145, 272

Cronin, J. R., \& Pizzarello, S. 1997, Science, 275, 951

Hitzfelder, S. J., Plass, G. N., \& Kattawar, G. W., 1976, Appl. Optics, 2489

Ivanoff, A., \& Waterman, T. H. 1958, J. of Marine Research, 16, 255

Mason, S. F. 1997, Nature ,389, 804

Smith, R. C., \& Baker, K. S. 1981, Appl. Optics, 20, 177

Waterman, T. H. 1954, Science, 120, 927

Waterman, T. H. 1955, Deep Sea Research Suppl., 3, 426

Wolstencroft, R. D. 1985, in IAU Symposium 112, The Search for Extraterrestrial Life (Dordrecht: Reidel), 171 